Table S1. KEGG categories and respective enriched genes regulated by Ang II at $\mathbf{3}$ hours interval

\section{KEGG category}

MAPK signaling pathway

Chemokine signaling pathway

Regulation of actin cytoskeleton

Tight junction

ErbB signaling pathway

Neurotrophin signaling pathway

Ubiquitin mediated proteolysis

Insulin signaling pathway

Chronic myeloid leukemia

Cell cycle

Glioma

VEGF signaling pathway

Gap junction

\section{Up-regulated Genes}

Mapk1, Cdc25b, Akt1, Kras, Map2k4, Mapkapk2, Mecom, Map2k5, Ppp3r1, Elk1, Rras, Ppp5c, Gna12

Shc3, Mapk1, Akt1, Kras, Cxcl10, Cxcl12, Gnai3, Prex1, Gnb2

Mapk1, Apc, Kras, Myl9, Rras, Fgd1, Pdgfc, Gna12, Git1

Akt1, Kras, Magi3, Gnai3, Myl9, Inadl, Rras, Pard6b, Llgl1

Shc3, Mapk1, Akt1, Kras, Map2k4, Elk1, Nck2

Shc3, Mapk1, Akt1, Kras, Mapkapk2, Map2k5, Sh2b1

Keap1, Ube2e3, Pias1, Mgrn1, Klhl13, Anapc10, LOC68922

Shc3, Mapk1, Akt1, Kras, Elk1, Rhoq

Shc3, Mapk1, Akt1, Kras, Mecom, E2f1

Cdc25b, Mcm5, Mcm6, Tfdp2, Anapc10, E2f1

Shc3, Mapk1, Akt1, Kras, E2f1

Mapk1, Akt1, Kras, Mapkapk2, Ppp3r1

Mapk1, Kras, Gnai3, Map2k5, Pdgfc
KEGG category

Protein processing in endoplasmic reticulum

Axon guidance

ECM-receptor interaction

TGF-beta signaling pathway

PPAR signaling pathway

mTOR signaling pathway

Notch signaling pathway

Aldosterone-regulated sodium

reabsorption

N-Glycan biosynthesis
Down-regulated Genes

Stub1, Ganab, Bag1, Tram1, Ckap4, Man1b1, Wfs1

Sema3c, Nfat5, Efn4, Ephb6, Unc5a, Slit1

Lama5, Cd47, Col1a1, Col5a3

Ep300, Gdf7, Ltbp1, Tgfb2

Dbi, Slc27a4, Scd4, Slc27a1

Rps6ka2, Ins1, Tsc2

Ep300, Ncstn, Psen2

Ins1, Prkca, Scnn1a

Ganab, Alg11, Man1b1 
Table S2. Biological processes (GO) and respective enriched genes regulated by Ang II at 3 hours interval

cell cycle

cell division

cell growth

protein transport

intracellular signal transduction

small GTPase mediated signal transduction

protein folding

protein ubiquitination /

polyubiquitination

actin cytoskeleton organization

MyD88-dependent toll-like

receptor signaling pathway

Wnt receptor signaling pathway

response to DNA damage stimulus

response to ionizing radiation
Apc, Ccng1, Nucks1, Rab11b, Sept9, Rgs2, Mapk1, Cdc25b, Cgref1, Mecom, Thap1, Specc1l, Ankrd54, Lzts, E2f1, Pdcd6ip

Cdc25b, Ccng1, Gnai3, Specc1l, LZts2, Pdcd6ip, Sept9 Akt1, Cxcl12, Map2k5, Taf9

Nutf2, Sec13, Atp6v1e1, Ipo9, Chchd4, Pex5, Chmp1a, Pdcd6ip, Exoc5, Ykt6, Atp6v1a, Rab11b Shc3, Mapk1, Akt1, Def8, Dnmbp, PRerx1, Blnk, Grb15,Sh2b1

Mapk1, Kras, Rnd2, Rras, Arl6, Rab11b, Rhoq, Gnai3, Gfm2, Gna12

Fkbp4, Ranbp2, Ppil4, Dnajc11, Ptges3, Grpel1, St13

Akt1, Mgrn1, Klhl13, Rnf187, Zfp364, March2, Ube2e3, Ube2v1, Mgrn1, Rnf187, LOC689226 Kras, Fhl3, Fgd1, Llgl1, Cap1, Rhoq

Mapk1, Map2k4, Mapkapk2, Ube2v1, Elk1

Wif1, Apc, Ldb1, Lzts2

Mapk1, Apc, Apbb1, Paxip1, Obfc2b

Cxcl10, Vcam1, Paxip1, Obfc2b
Biological Process

ion transport

brain development

protein transport

heart development

negative regulation of cell proliferation

negative regulation of apoptosis

response to hypoxia

axon guidance

protein folding

negative regulation of cell growth cell migration

\section{Down- regulated Genes}

Steap3, Tmem37, Scnn1a, Kcnh3, Kcnk3, Gabrd, Slc22a18, Cacna1i, Slc22a23, Slc12a6

Ptprg, Dbi, Cdk5rap1, Nkx2-1, Cdk5rap2, Kcnk3, Bcr, Slc7a11, Slit1, Hrh3

Steap3, Aagab, Dnm2, Mcoln1, Cox18, Apoc2, Col1a1, Lca5, Tram1, Psen

Rps6ka2, Sox9, Ep300, Gja1, Tsc2, Oxt, Sema3c, Sox18, Tgfb2

Sox9, Gja1, Grm4, Igfbp3, Prkca, Tsc2, Serpine2, S2f7, Tgfbp2

Sox9, Grm4, Bag1, Cln8, Tex11, Psen2, Tgfb2, Wfs1

Ep300, Pnmt, Tsc2, Kcnk3, Agtrap, Adsl, Psen2, Tgfbp2

Gdf7, Nkx2-1, Neurog2, Unc5a, Slit1, Tgfb2

Ganab, Bag1, RGD1565752, Dnajc17, Fxbp10, Man1b1

Dnajc2, Serpine2, Ndufa13, Slit1, Tgfb2

Lama5, Cd47, Lamb1, Mdk, Tgfb2 
Table S3. KEGG categories and respective enriched genes regulated by Ang II at 6 hours interval

Cell cycle

Pathways in cancer

RNA transport

MAPK signaling pathway

Focal adhesion

Insulin signaling pathway

Protein processing in endoplasmic reticulum

Spliceosome

Ubiquitin mediated proteolysis

Proteasome

ErbB signaling pathway

Neurotrophin signaling pathway p53 signaling pathway

Adherens junction

Chronic myeloid leukemia
Chek2, Ccna2, Bub1b, Ccnb1, Ywhaq, Skp2, Smc3, Mcm4, Hdac1, Anapc1, Stag2, Cdc7, MGC112830, Anapc10, Cdk2, Cdc26, Cdkn2c, Cdkn2d, Cdc20

Mapk1, Smo, Ctbp1, Skp2, Hdac1, Pias1, Stk36, Col4a2, Cdk2, Tcf7, Mapk9, Ccdc6, Grb2, Mlh2, Sos2

Nup107, Eif4e, Eif4a1, Thoc3, Ncbp1, Pabpc4, Sumo1, RGD1306195, Nup205, Eif4e2, Gemin7, Sumo2, Thoc4 Mapk1, Mapk4k3, Hspa8, Hspa1l, Ntf4, Map2k4, Pak2, Elk1, Mapk9, Chp, Grb2, Sos2

Mapk1, Capn2, Pak2, Vcl, Col4a2, Elk1, Vasp, Itga8, Mapk9, Grb2, Sos2

Mapk1, Eif4e, Elk1, Eif4e2, Prkag2, Mapk9, Calm2, Grb2, Sos2

Nploc4, Hspa8, Hspa1I, Hsph1, Capn2, Man1a2, Ube2j2, Mapk9, Eif2ak2

Hspa8, Hspa1I, Thoc3, Snrpd1, Ncbp1, Ism6, Pcbp1, PIrg1, Thoc4

Skp2, Ube2q1, Ube2j2, Pias1, Anapc1, Anapc10, Ube2l3, Cdc26, Cdc20

Psme3, Pomp, Psma1, Psmd14

Mapk1, Map2k4, Pak2, Abl2, Elk1, Mapk9, Grb2, Sos2

Mapk1, Ywhaq, Ntf4, Nfkbie, Mapk9, Calm2, Grb2, Sos2

Chek2, Ccnb1, Ei24, Cdk2, Zmat3

Mapk1, Yes1, Vcl, Ctnnd1, Tcf7

Mapk1, Ctbp1, Hdac1, Grb2, Sos2
KEGG category

Parkinson's disease

Mineral absorption

Cytosolic DNA-sensing pathway

Pantothenate and CoA biosynthesis

Protein export

Riboflavin metabolism

\section{Down-regulated Genes}

Cox6a2, Cyc2, Ndufs2, Ube2g2, Ube216, Ndufa12, Ndufa6, Ndufv3, Ndufa3

Slc31a1, Slc39a3, Slc26a6, Hmox2

Ripk3, Ifna4, RGD1562552, Polr3g

Coasy, Ppcs, Enpp3

Srp19, Srpr, Sec61g

Acp5, Enpp3 
Table S4. Biological processes (GO) and respective enriched genes regulated by Ang II at 6 hours interval

\section{Biological Process}

protein phosphorylation

negative regulation of apoptosis

positive regulation of apoptosis

proteasomal ubiquitin-dependent protein catabolic process

negative regulation of cell proliferation

cell cycle

cell division

nervous system development

DNA repair

actin cytoskeleton organization

DNA replication

protein ubiquitination

mitosis

RNA splicing
Up-regulated Genes

Chek2, Nek2, Wnk1, Mapk1, Cdk5, Map4k3, Camk1, Cdk12, YEs1, Ccnb1, Dyrk1a, Map2k4, Pak2, Sgk1, Tbk1, Lrrk2, Stk36, Abl2, Pxk, Mst4, Nek6, Spag9, Cdc7, Vrk1, Cdk2, Pik3r4, Mapk9, Eif2ak2

Dffa, Il6r, Serbp1, Glp1r, Smo, Fnta, Accn1, Fignl1, Gata6, Hells, Htt, Skp2, Sgk1, Hdac1, Apbb2, Serpinb9, Pea15a, Taf9, Rtn4, Twist1

Dffa, Cdk5, Aldh1a3, Map2k4, Gata6, Skp2, Ing4, Sav1, Apbb2, RGD1309922, Top2a, Mapk9, Eif2ak2

Bub1b, Ccnb1, Psme3, Ube2v2, Psmd5, Psma1, Irrk2, Sumo1, Fbxl5, Psmd14, Anapc1, Anapc10, Trim13, Cdc26, Taf9, Cdc20, Ppp2r5c, Gipc1

Phb2, Cd9, Igfbp5, Tinf2, Ing4, Hdc1, Sav1, Lrrk2, Brip1, Cdkn2c, Eif2ak2, Dlc1, Slc9a3r1, Mfn2, Inppl1, Ppp2r5c

Ccna2, Clasp2, Mapk1, Cdk5, Kif2c, Smc3, Arhgef2, Nek6, Spast, Cdk2, Cdc26, Calm2, Cdc20, Mlh1

Ccna2, Clasp2, Cdk5, Kif2c, Ccnb1, Smc3, Arhgef2, nek6, Top2a, Spast, Cdk2, Cdc26, Cdc20, Top1

Myo1b, Camk1, Dyrk1a, Accn1, Ube2v2, Dpf3, Lrrk2, Ophn1, Spast, Kctd11, LOC606294, Rtn4, Nrn1, Ppp1r9b

Polk, Wrnip1, Ube2v2, Polq, Fbxo18, Rbbp8, Fto, Smc3, Pole, Rev3l, Recql, Rnf168, Mlh1, Parg

Cdk5, Mk1, Sipa1l1, Coro1b, Capzb, Abl2, Ophn1, Ehd2, Vasp, Dlc1, Slc9a3r1, Cap1, Capza1

Wrnip1, Map2k4, Polq, Mcm4, Ing4, Pole, Rev3l, Recql, Cdc7, Cdk2, Helb, Top1

Rnf34, Skp2, Rnf2, Fbxl5, Anapc1, Anapc10, Rfws3, Ube2l3, Trim13, Cdc26, Rnf168, Ccnb1, Psme3, Psmd5, Psma1, Irrk2, Psmd14, Tbc1d7, Cdc20

Clasp2, Kif2c, Ccnb1, Jtb, Smc3, Arhgef2, Nek6, Cdk2, Cdc26, Cdc20

Cdk13, Snrpd1, Cd2bp2, Ncbp1, Hnrnpa2b1, Srek1, PIrg1, Thoc4, Polr2f 


\author{
apoptosis \\ protein transport \\ intracellular protein transport \\ protein ubiquitination \\ ubiquitin-dependent protein \\ catabolic process \\ response to oxidative stress \\ vesicle-mediated transport \\ protein targeting \\ positive regulation of cell \\ migration \\ cytoskeleton organization
}

Psmd9, Acsbg1, Ripk3, Prkcb, Cycs, Aqp2, Ncstn, Psmg2, Actc1, Fastk, Arhgef4, Ciapin1, Plekhf1, Trim39, Traf2, Aen, Itgb3bp, Scrip, Pdcd2, Dapk3, Aifm1, Plg

Pgap2, Arfgap1, Abra, Vps36, Snx17, Rabif, Chmp2b,

Chmp7, Efcab4b, Arfgap3, Gorasp1, Vps33b, Pex14, Snap23, Scamp2, Vps25, LOC681989, Fxc1, Rab27b, Sec31a

Vamp1, Abra, Ipo4, Napg, Ipo7, Srpr, Snupn, Tom112, Tom1, Sar1a, Xpo5, Scamp2, LOC683402

Zfp91, Ube2g2, Rnf146, Uhrf2, Traf2, Tri32, KIhl21, Cbll1, Mdm2, Ube3a, Herc6, Anapc13

Usp2, Usp4, Usp15, Rnf146, Uhrf2, Trim32, Mdm2, Ube3a, Herc6, Usp48

Abcc1, Coq7, Cycs, Ndufs2, Ndufa12, Ppp1r15b, Lias, Ndufa6, Hmox2

Arfgap1, Vamp1, Sar1a, Vamp4, Arfgap3, Vps33b, Snap23, LOC683402, Sec31a

Optn, Srp19, Strada, Mdm2, Srpr, Xpo5, Pmpcb, Pex14, Fxc1

Abcc1, Acp5, Trim32, Cbll1, Srpx2, Cxcl16, Ager, Vegfa

Cdc42bpa, Uxt, Bbs4, Tekt4, Tubgcp2, MAST1, Ablim2, Trpv4, Rhoq 
Table S5. KEGG categories and respective enriched genes regulated by Ang II via AT1 receptor at 3 hours interval

\begin{tabular}{|c|c|}
\hline KEGG category & Up-regulated Genes \\
\hline Metabolic pathways & $\begin{array}{l}\text { Ndst2, Hsd17b1, B3gnt1, Polrf3f, Etnk1, Rdh12, Chpf, } \\
\text { Pigu, Alg11, Alg9, Rfk, Rpe }\end{array}$ \\
\hline Lysosome & Mcoln1, Aga, Abca2 \\
\hline Acute myeloid leukemia & Myc, Rps6kb2 \\
\hline Steroid hormone biosynthesis & Sts, Hsd17b1 \\
\hline N-Glycan biosynthesis & Alg11, Alg9 \\
\hline KEGG category & Down-regulated Genes \\
\hline Cell cycle & Chek2, Cdc25b, Rp53, Anapc5, Mcm5, Smc1a \\
\hline p53 signaling pathway & Chek2, Tp53, Tsc2 \\
\hline $\begin{array}{l}\text { Amino sugar and nucleotide sugar } \\
\text { metabolism }\end{array}$ & Gale, Amdhd2, Gmppa \\
\hline beta-Alanine metabolism & Acadm, Aldh2 \\
\hline Propanoate metabolism & Acadm, Aldh2 \\
\hline $\begin{array}{l}\text { Glycine, serine and threonine } \\
\text { metabolism }\end{array}$ & Shmt2, Gcat \\
\hline $\begin{array}{l}\text { Vasopressin-regulated water } \\
\text { reabsorption }\end{array}$ & Dctn6, Dynll1 \\
\hline DNA replication & Mcm5, Pola1 \\
\hline
\end{tabular}


Table S6. Biological processes (GO) and respective enriched genes regulated by Ang II via AT1 receptor at 3 hours interval

\begin{tabular}{|c|c|}
\hline Biological Process (GO) & Up-regulated Genes \\
\hline protein transport & Lman1, Unc50, Mcoln1, Tram1, Atg9a \\
\hline positive regulation of apoptosis & Jak2, Myc, TIr4, Stk4 \\
\hline steroid metabolic process & Lcat, Sts, Hsd17b1, Scap \\
\hline inflammatory response & $\begin{array}{c}\text { Tnfrsf1b, Tnfrsf4, Tlr4, Jak, Ccl24, Zyx, } \\
\text { Cd276 }\end{array}$ \\
\hline tumor necrosis factor-mediated signaling pathway & Tnfrsf1b, Jak2, Tnfrsf4 \\
\hline positive regulation of cell migration & Jak2, Ccl24, Lamb1 \\
\hline intracellular protein kinase cascade & Jak2, Stk4 \\
\hline Biological Process (GO) & Down-regulated Genes \\
\hline cell cycle / division and mitosis & $\begin{array}{c}\text { Cdc25b, Avpi1, Ppp1c, Anapc5, Kifc1, } \\
\text { Mtus1, RGD1306730, Smc1a, Arl2, } \\
\text { Mlh1 }\end{array}$ \\
\hline regulation of cell cycle & Cdc37, Tp53, Tsc2, Bop1 \\
\hline DNA repair & Fbxo18, Kctd13, Smc1a, Mlh1, Pola1 \\
\hline carbohydrate metabolic process & Gale, Ppp1c, Amdhd2, Chst5 \\
\hline protein homooligomerization & Acadm, Tsc2, Kctd13, Shmt2 \\
\hline cell migration & Bcar1, Kctd13, Gsk3a, Bambi \\
\hline negative regulation of signal transduction & Tsc2, Erbb3, Gsk3a, Rfng \\
\hline $\begin{array}{l}\text { DNA damage response, signal transduction } \\
\text { resulting in induction of apoptosis }\end{array}$ & Chek2, Tp53, Mlh1 \\
\hline circadian rhythm & Nfil3, Erbb3, Arntl \\
\hline insulin receptor signaling pathway & Eif4ebp1, Bcar1, Gsk3a \\
\hline $\begin{array}{l}\text { anaphase-promoting complex-dependent proteasomal } \\
\text { ubiquitin-dependent protein catabolic process }\end{array}$ & Psmd9, Psme3, Anapc5 \\
\hline neuroblast proliferation & Tp53, Racgap1, Sox2 \\
\hline TOR signaling cascade & Eif4ebp1, Tsc2 \\
\hline $\begin{array}{l}\text { positive regulation of epithelial } \\
\text { to mesenchymal transition }\end{array}$ & Wwtr1, Bambi \\
\hline cytokinesis & Incenp, Racgap1 \\
\hline Rho protein signal transduction & Kctd13, Cdc42ep1 \\
\hline negative regulation of Wnt receptor signaling pathway & Tsc2, Sox2 \\
\hline
\end{tabular}


Table S7. KEGG categories and respective enriched genes regulated by Ang II via AT1 receptor at 6 hours interval

\begin{tabular}{|c|c|}
\hline KEGG category & Up-regulated Genes \\
\hline RNA transport & $\begin{array}{c}\text { Eif4e, Ube2i, Pop4, Nup35, Gemin6, } \\
\text { Pigz, Gemin2 }\end{array}$ \\
\hline Cell cycle & $\begin{array}{c}\text { Chek1, Ccne1, Cdc45, Mad2I1, } \\
\text { Gadd45b }\end{array}$ \\
\hline Peroxisome & Pxmp4, Pex19, Eci2, Pex11b \\
\hline Pyrimidine metabolism & Uck1, Cmpk2, Polr3k, Polr3gl \\
\hline p53 signaling pathway & Chek1, Ccne1, Gadd45b \\
\hline Proteasome & Psmb9, Psbm4, Psmb1 \\
\hline Riboflavin metabolism & Acp5, Acp6 \\
\hline Homologous recombination & Rad54b, Rpa2 \\
\hline RNA polymerase & Polr3k, Polr3gl \\
\hline KEGG category & Down-regulated Genes \\
\hline Focal adhesion & $\begin{array}{l}\text { Bcar1, Ptk2, Itgb4, Pak1, Myl9, Flnb, } \\
\text { Itgb6, Actn4, Vegfa }\end{array}$ \\
\hline Regulation of actin cytoskeleton & $\begin{array}{c}\text { Bcar1, Ptk2, Itgb4, Pak1, Myl9, Itgb6, } \\
\text { Iqgap1, Actn4 }\end{array}$ \\
\hline Chemokine signaling pathway & Plcb1, Stat3, Bcar1, Ptk2, Pak1, Gngt2 \\
\hline Lysosome & Ctsh, Cdig2, Gga2, Ctsa, Abcb9 \\
\hline Axon guidance & Ptk2, Pak1, Sema3c, Sema3f, Slit2 \\
\hline PPAR signaling pathway & Acsl1, Scd4, LOC681458, Gk \\
\hline Adherens junction & Smad2, Ssx2ip, Iqgap1, Actn4 \\
\hline Aminoacyl-tRNA biosynthesis & Aars, Farsb, Wars \\
\hline Biosynthesis of unsaturated fatty acids & Scd4, LOC681458 \\
\hline Vitamin digestion and absorption & Ten2, Cubn \\
\hline
\end{tabular}


Table S8. Biological processes (GO) and respective enriched genes regulated by Ang II via AT1 receptor at 6 hours interval

\begin{tabular}{|c|c|}
\hline Biological Process (GO) & Up-regulated Genes \\
\hline protein ubiquitination & $\begin{array}{l}\text { Psmb9, Arrb2, Ube2i, Ube2t, Psmb4, Rnf168, } \\
\text { Psmb1, Arrb2, Mad2I1, Birc5 }\end{array}$ \\
\hline $\begin{array}{l}\text { proteasomal ubiquitin-dependent } \\
\text { protein catabolic process }\end{array}$ & $\begin{array}{l}\text { Psmb9, Arrb2, Ube2i, Mad2I1, Jkamp, Psmb4, } \\
\text { Psmb1 }\end{array}$ \\
\hline mitotic cell cycle & Eif4e, Psmb9, Mad2I1, Psmb4, Birc5, Psmb1 \\
\hline DNA repair & Chek1, Gen1, Alkbh2, Rpa2, Rnf168 \\
\hline regulation of cell growth & Cd44, Exosc9, Ccdc85b, Ulk1, Zfp639 \\
\hline cell division & Ube2i, Ccne1, Cdc45, Spast, Birc5 \\
\hline cellular process involved in reproduction & Sep15, Arrb2, Nkx2-1, Syt6, Zfp639 \\
\hline cell cycle process & Psmb9, Mad2I1, Psmb4, Birc5, Psmb1 \\
\hline response to peptide hormone stimulus & Prss8, Aqp2, Prlh, Birc5 \\
\hline Biological Process (GO) & Down-regulated Genes \\
\hline signal transduction & $\begin{array}{c}\text { Chd4, Cdh13, Grin2c, Ngfr, Il1rl1, Itpr3, Xpr1, } \\
\text { Aars, Srebf2, Arhgap28, Fert2, Scara5, Ngf, } \\
\text { Iqgap1, Vapb, Arhgap10 }\end{array}$ \\
\hline cell differentiation & $\begin{array}{l}\text { Egr2, KIf4, Sox9, Ngfr, Plcb1, Ptk2, Jag1, Clip3, } \\
\text { Cd276, Slit2, Zmiz1, Sohlh1, Srf, Ascl1, Dhh }\end{array}$ \\
\hline response to organic substance & $\begin{array}{c}\text { Abcc4, Cdh13, Stat3, Acsl1, Fosl1, Ptk2, Sdc2, } \\
\text { Smad2, Pak1, Srebf2, Ngf, Slit2, Pea15a, Hspd1, } \\
\text { GK }\end{array}$ \\
\hline regulation of cell proliferation & $\begin{array}{c}\text { Nrg1, Sox9, Abcc4, Cdh13, Ngfr, Irs1, Ptk2, Ngf, } \\
\text { Zmiz1, Sox4, Apln, Nolc1, Vegfa, Nr2f2, KIf4, } \\
\text { Jag1 }\end{array}$ \\
\hline negative regulation of apoptosis & $\begin{array}{c}\text { Nrg1, Sox9, Cdh13, Ngfr, Dnajb9, Ptk2, Lrp1, Ngf, } \\
\text { Pea15a, Sox4, Hspd1, Ascl1, Vegfa }\end{array}$ \\
\hline response to stress & $\begin{array}{l}\text { Adh5, Ubqln1, Dnajb9, Sdc2, Aars, Pak1, Scara5, } \\
\text { Sox4, Vapb, Actn4, Hspd1, Gk, Vegfa }\end{array}$ \\
\hline regulation of signal transduction & $\begin{array}{c}\text { KIf4, Avpi1, Cdh13, Ngfr, Irs1, Jag1, Smad2, Pak1, } \\
\text { Ngf, Sulf2, Slit2, Ascl1, Vegfa }\end{array}$ \\
\hline cell surface receptor linked signaling pathway & $\begin{array}{c}\text { Plcb1, Spn, Stat3, Acsl1, Bcar1, Gfra1, Il1rap, } \\
\text { ॥1rl1, Itgb4, Fcgr2b, Itgb6, Slit2 }\end{array}$ \\
\hline cell adhesion & $\begin{array}{l}\text { Nrg1, Cdh13, Bcar1, Ptk2, Itgb4, Fert2, Pcdh9, } \\
\text { Ssx2ip, Itgb6, Omd }\end{array}$ \\
\hline
\end{tabular}


Table S9. KEGG categories and respective enriched genes regulated by Ang II via AT2 receptor at 3 hours interval

\begin{tabular}{|c|c|}
\hline KEGG category & Up-regulated Genes \\
\hline Purine metabolism & Gucy1b2, Pfas, Polr2i \\
\hline Steroid hormone biosynthesis & Ugt1a6, Сyp1b1 \\
\hline Retinol metabolism & Ugt1a6, Dhrs3 \\
\hline $\begin{array}{l}\text { Metabolism of xenobiotics } \\
\text { by cytochrome P450 }\end{array}$ & Ugt1a6, Сyp1b1 \\
\hline Drug metabolism - other enzymes & Ugt1a6, Ces2a \\
\hline N-Glycan biosynthesis & Ganab, Man1c1 \\
\hline KEGG category & Down-regulated Genes \\
\hline Pathways in cancer & $\begin{array}{c}\text { Pik3ca, Itgb1, Myc, Prkca, Fgf9, Axin2, Hdac1, } \\
\text { Cbl, Birc5, Sos2 }\end{array}$ \\
\hline MAPK signaling pathway & $\begin{array}{c}\text { Dusp6, Myc, Prkca, Fgf9, Rasa1, Pak2, Ppp3r1, } \\
\text { Rras, Sos2 }\end{array}$ \\
\hline Regulation of actin cytoskeleton & $\begin{array}{c}\text { Ppp1r12a, Pik3ca, Itgb1, Fgf9, Pak2, Itga4, } \\
\text { Rras, Sos2 }\end{array}$ \\
\hline ErbB signaling pathway & $\begin{array}{c}\text { Pik3ca, Myc, Prkca, Hbegf, Areg, Pak2, Cbl, } \\
\text { Sos2 }\end{array}$ \\
\hline Focal adhesion & $\begin{array}{l}\text { Ppp1r12a, Pik3ca, Itgb1, Prkca, Pak2, Itga4, } \\
\text { Sos2 }\end{array}$ \\
\hline Axon guidance & Itgb1, Rasa1, Pak2, Ppp3r1 \\
\hline VEGF signaling pathway & Pik3ca, Prkca, Ppp3r1 \\
\hline
\end{tabular}


Table S10. Biological processes (GO) and respective enriched genes regulated by Ang II via AT 2 receptor at 3 hours interval

\begin{tabular}{|c|c|}
\hline Biological process (GO) & Up-regulated Genes \\
\hline cell differentiation & $\begin{array}{c}\text { Igfbp3, Gpsm1, Ggn, Sox18, Lrp6, NrOb1, } \\
\text { Mdk }\end{array}$ \\
\hline angiogenesis & Ecm1, Cyp1b1, Ccl24, Sox18, Pnpla6 \\
\hline response to peptide hormone stimulus & Ugt1a6, Pnmt, Lrp6, Nr0b1 \\
\hline embryo development & Foxn3, Ccno, Foxj2, Fzd2 \\
\hline $\begin{array}{l}\text { regulation of sequence-specific DNA binding } \\
\text { transcription factor activity }\end{array}$ & Lrp6, Foxn3, Foxj2, NrOb1 \\
\hline vasculature development & Cypb1, Sox18, Fzd2 \\
\hline canonical Wnt receptor signaling pathway & Lrp6, Fzd2, Bambi \\
\hline Biological process $(\mathrm{GO})$ & Down-regulated Genes \\
\hline cell differentiation & $\begin{array}{c}\text { Dusp6, Bzw2, Fgf9, Snai2, Rasa1, Axin2, } \\
\text { Areg, Ppp3r1, Rora, Runx2, Sh2b3, Fh12, } \\
\text { Ctgf, Dhh }\end{array}$ \\
\hline negative regulation of apoptosis & $\begin{array}{c}\text { Pik3ca, Myc, Serbp1, Rasa1, Zc3hc1, Hdac1, } \\
\text { Api5, Pea15a, Cbl, Birc5, Pim3 }\end{array}$ \\
\hline positive regulation of cell proliferation & $\begin{array}{c}\text { Itgb1, Myc, Prkca, Hbegf, Fgf9, Areg, Hdac1, } \\
\text { Etv5, Aggf1, Runx2, Ctgf }\end{array}$ \\
\hline ossification & $\begin{array}{c}\text { Mmp13, Fgf9, Snai2, Axin2, Areg, Impad1, } \\
\text { Nab2, Runx2, Fhl2, Ctgf }\end{array}$ \\
\hline negative regulation of cell proliferation & $\begin{array}{c}\text { Gtpbp4, Itgb1, Prkca, Emd, Eif2ak1, Axin2, } \\
\text { Hdac1, Eif2ak2 }\end{array}$ \\
\hline cell adhesion & $\begin{array}{c}\text { Cdh17, Itgb1, Rasa1, Tnfrsf12a, Aggf1, Itga4, } \\
\text { Ctgf, Cdhr1 }\end{array}$ \\
\hline canonical Wnt receptor signaling pathway & Emd, Fgf9, Snai2, Axin2, Hdac1, Lats2, Cyld \\
\hline regulation of signal transduction & $\begin{array}{c}\text { Dusp6, Zfp91, Prkca, Fgf9, Slc7a1, Hdac1, } \\
\text { Runx2 }\end{array}$ \\
\hline regulation of cell proliferation & Myc, Prkca, Hbegf, Emd, Fgf9, Etv5, Eif2ak2 \\
\hline aging & Lonp1, Prkca, Eno3, Canx, Ctgf, Tfrc \\
\hline dephosphorylation & $\begin{array}{l}\text { Dusp6, Ppp1r12a, Acp2, Ppp3r1, Impad1, } \\
\text { Pdp1 }\end{array}$ \\
\hline regulation of mitotic cell cycle & Myc, Cyld, Brd4, Birc5, Pim3, LOC690728 \\
\hline
\end{tabular}


Table S11. KEGG categories and respective enriched genes regulated by Ang II via AT2 receptor at 6 hours interval

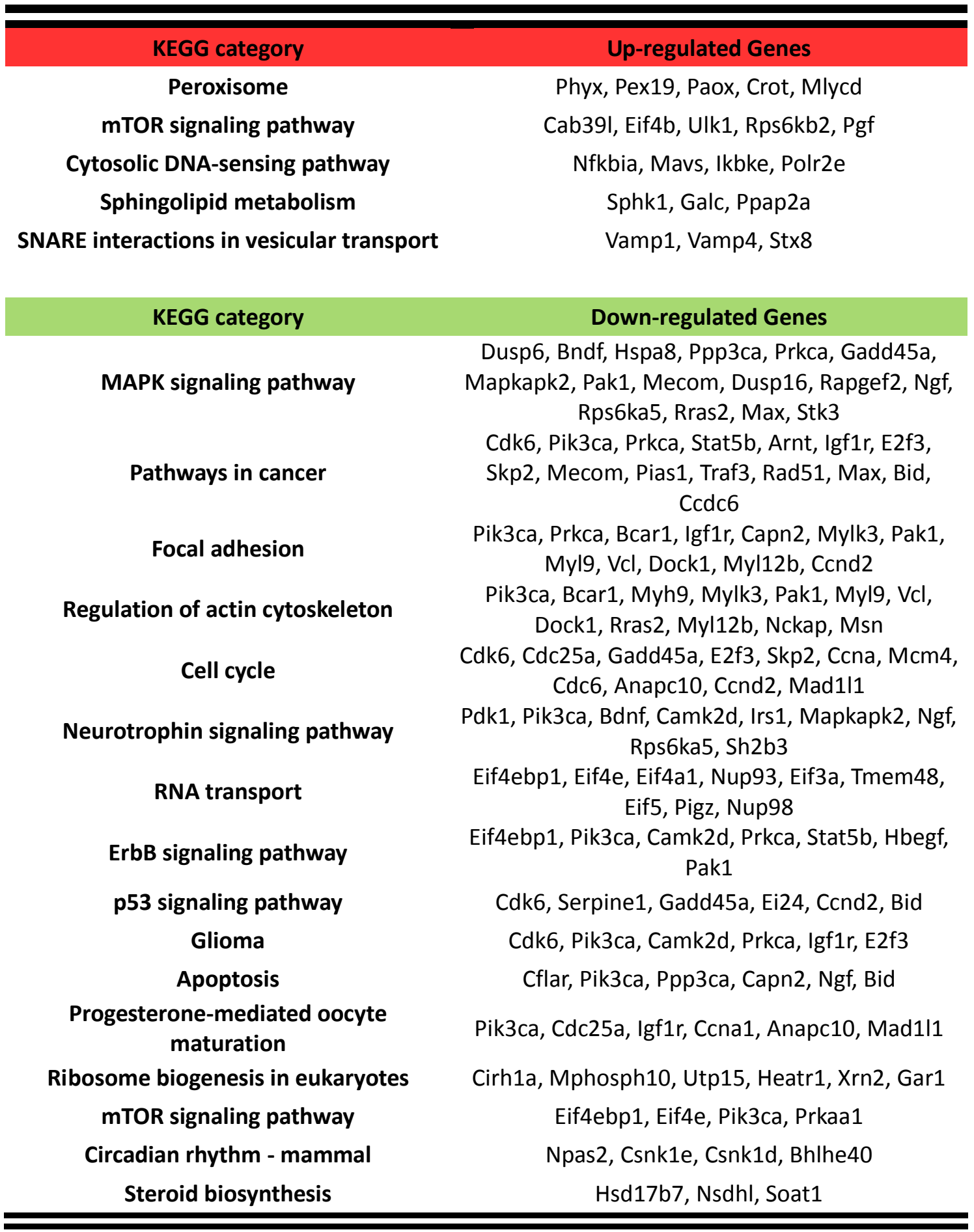


Table S12. Biological processes (GO) and respective enriched genes regulated by Ang II via AT2 receptor at 6 hours interval

\begin{tabular}{cc}
\hline $\begin{array}{c}\text { Biological Process (GO) } \\
\text { positive regulation of } \\
\text { cell proliferation } \\
\text { protein transport }\end{array}$ & $\begin{array}{c}\text { Up-regulated Genes } \\
\text { Sphk1, Il11, Casr, Erbb2, Stat5a, Ccnb1, Rogdi, Cd47, } \\
\text { Myd88, Tbx18, Sox4, Cyba }\end{array}$ \\
brain development & $\begin{array}{r}\text { Dnajc14, Exoc3, Dnm2, Mcoln1, Nutf2, Tmed3, Duoxa1, } \\
\text { Chmp1a, Gorasp1, Psen2, Sec16b }\end{array}$ \\
Stxbp3, Sphk1, Dmbx1, Tmem57, Bbc3, Fzd1, Tulp3, \\
Cdk5rap3, Sharpin, Synj2
\end{tabular}

\begin{tabular}{|c|c|}
\hline Biological Process (GO) & Down-regulated Genes \\
\hline protein phosphorylation & $\begin{array}{c}\text { Nek2, Cdk6, Pdk1, Pik3ca, Cdk12, Prkca, Igf1r, Mapkpk2, } \\
\text { Mylk3, Pak1, Lmtk2, Lats2, Mast4, Pdik1l, Rps6ka5, MSt4, } \\
\text { Nek7, Vrk2, Riok3, Mdfic, Epha2, Bmx, Gsk3a, Csnk1e, } \\
\text { Csnk1d, Stk3, Prkaa1, Hipk2, Plk2 }\end{array}$ \\
\hline signal transduction & $\begin{array}{l}\text { Stat5b, Il1rl1, Ahr, Rtkn, Ngf, Asap1, Traf3, Siva1, Syde1, } \\
\text { Rras2, Stard13, Gsk3a, Pdpn, Ramp2, Arhgap17, Stk3, } \\
\text { Arhgap10, Akap12, Gnb5, Plk2, Hmga2, Bpm7 }\end{array}$ \\
\hline apoptosis & $\begin{array}{c}\text { Slah2, Hip1, Ahr, Nlrp3, Rtkn, Ngf, Fam82a, Nlrp1a, Bag4, } \\
\text { Aen, Siva1, Pea15a, Epha2, Dap, Bid, Stk3 }\end{array}$ \\
\hline protein transport & $\begin{array}{l}\text { Stxb1, Myh9, Abra, Nup93, Lmtk2, Ipo9, RUfy1, Tmem48, } \\
\text { Rab6b, Pex14, Timm10, Vps45, Arf2, Nup98, Sec31a }\end{array}$ \\
\hline negative regulation of apoptosis & $\begin{array}{c}\text { Cflar, Pik3ca, Bdnf, Stat5b, Igf1r, Skp2, Btg2, Rtkn, Ngf, } \\
\text { Ndufaf4, Siva1, Pea15a, Hipk3, Rtn4, Cat, Serpine1, } \\
\text { Accn1, Cd44, Nes, Fignl1, Btg2, Ung, Tfap4, Wfs1, Bmp7 }\end{array}$ \\
\hline $\begin{array}{l}\text { response to organic } \\
\text { cyclic compound }\end{array}$ & $\begin{array}{l}\text { Dusp6, Bdnf, Eno2, Prkca, Cd44, Ahr, Homer1, Btg2, Ngf, } \\
\text { Atf1, Ctgf, Ccdnd2 }\end{array}$ \\
\hline regulation of cell proliferation & $\begin{array}{c}\text { Cdk6, Serpine1, Prkca, Stat5b, Hbgef, Plcd3, Skp2, } \\
\text { Mecom, Atp2b1, Mfn2, Bid, Rbpj }\end{array}$ \\
\hline cell cycle & $\begin{array}{c}\text { Cdk6, Siah2, Cdc25a, Ppp6c, Ahr, Spc25, Ccdc99, Thap1, } \\
\text { Specc1l, Pdpn, Rgs2 }\end{array}$ \\
\hline
\end{tabular}


response to estrogen stimulus

DNA replication

regulation of apoptosis

nervous system development

response to stress

cell division
Eno2, Serpine1, Prkca, Stat5b, Ahr, Rbbp5, Ramp2, Ctgf, Cond2, Bid, Bmp7

Rfc2, Cdc25a, Kctd13, Mcm4, Clspn, Pole, Blm, Cdc6, Ctgf, Nup98

Cflar, Hip1, Mllt11, Deptor, Mst4, Traf3, Pea15a, Bid, Wfs1 Bdnf, Hbgef, Nes, Kat2a, Fnbp1l, Epha2, Slc7a5, Pdpn, Bhlhe40, Rtn4

Bdnf, Cat, Hspa8, Serpine1, ppp3ca, Ahr, Homer1, Rps6ka5, Pdpn, Ctgf

Cdc5a, Ccna1, Spc25, Ccdc99, Top2a, Cdc6, Specc1l, Cond2 